\title{
Analysis of interaction between the apicomplexan protozoan Toxoplasma gondii and host cells using label-free Raman Spectroscopy
}

\author{
Abida Naemat ${ }^{a}$, Hany M. Elsheikha ${ }^{b}$, Alaa Al-sandaqchi ${ }^{b}$, Kenny Kong ${ }^{a}$, Adrian \\ Ghita $^{a}$ and Ioan Notingher ${ }^{a^{*}}$ \\ ${ }^{a}$ School of Physics and Astronomy, University of Nottingham, Nottingham, NG7 2RD, \\ $U K$ \\ ${ }^{\mathrm{b}}$ Faculty of Medicine and Health Sciences, School of Veterinary Medicine and Science, \\ University of Nottingham, Sutton Bonington Campus, Leicestershire, LE12 5RD, UK \\ * Corresponding author: \\ Tel. No.: +44-01159515172 Fax No.: +44-01159515180 \\ E-mail address: ioan.notingher@nottingham.ac.uk
}

\begin{abstract}
Label-free imaging using Raman micro-spectroscopy (RMS) was used to characterize the spatio-temporal molecular changes of $T$. gondii tachyzoites and their host cell microenvironment. Raman spectral maps were recorded from isolated $T$. gondii tachyzoites and T. gondii-infected human retinal cells at $6 \mathrm{hr}, 24 \mathrm{hr}$ and $48 \mathrm{hr}$ postinfection. Principal component analysis (PCA) of the Raman spectra of paraformaldehyde-fixed infected cells indicated a significant increase in the amount of lipids and proteins in the $T$. gondii tachyzoites as the infection progresses within host cells. These results were confirmed by experiments carried out on live $T$. gondii-infected cells and were correlated with an increase in the concentration of proteins and lipids required for the replication of this intracellular pathogen. These findings demonstrate the potential of RMS to characterize time- and spatially-dependent molecular interactions between intracellular pathogens and the host cells. Such information may be useful for discovery of pharmacological targets or screening compounds with potential neuroprotective activity for eminent effects of changes in brain infection control practices.
\end{abstract}




\section{Introduction}

One of the most important pathogens affecting the central nervous system and causing serious neurological disease in humans and animals is the obligate intracellular apicomplexan protozoan parasite Toxoplasma gondii. ${ }^{1}$ Even though most $T$. gondii infections are benign, this pathogen can infect virtually any nucleated cell in-vitro and is a significant cause of morbidity during pregnancy and in immunosuppressed patients as well as visual impairment. ${ }^{2}$ The parasite's remarkable ability to infect a broad range of hosts and cell types is due, in part, to the diverse arsenal of effectors it secretes into the host cell. For better understanding of the pathogenesis of $T$. gondii it is critical to identify the chemical molecules that mediate the interaction between this organism and the host cell. However, the host-pathogen interaction involves highly orchestrated, complex timedependent molecular interactions. Extracting quantitative information without disturbing the cell, while preserving molecular specificity and high spatial resolution remains a grand challenge when imaging individual living cells.

Imaging techniques have contributed significantly to the understanding of the $T$. gondii biology. ${ }^{3}$ However, although currently available conventional imaging techniques provided a considerable amount of information they are still insufficient to provide an improved understanding of the precise molecular mechanism of disease caused by this ubiquitous parasite. Most conventional imaging techniques require labelling, fixation, lysis or other invasive procedures. Also, these techniques provide only single time-shots and cannot provide insight into discrete and dynamic molecular events in living cells.

Recent label-free molecular imaging techniques using vibrational spectroscopy, such as Raman micro-spectroscopy (RMS), have contributed in various fields of life sciences. RMS is a well-established analytical technique that enables label-free chemical analysis of individual cells and bacteria with sub-micrometric spatial resolution. ${ }^{4-8}$ RMS was also used for time-course experiments on individual live cells revealing molecular processes not attainable with other imaging. ${ }^{9-13}$ The integration of environmental chambers with inverted Raman microscopes can allow time- and spatially-resolved molecular studies of cellular processes spanning days and weeks, such as stem cell differentiation. 14,15 Nevertheless, RMS has been used only in few studies to characterize molecular details associated with host-parasite interaction. For example, RMS has been utilized to study the protozoan pathogen Plasmodium falciparum, the agent of human malaria. ${ }^{16,17}$ Also, we previously developed a new method of measuring spatially-resolved Raman spectra of human cells by selective-sampling Raman microscopy and employed this method for the detection of the protozoan Neospora caninum in human blood-brain-barrier endothelial cells. ${ }^{18}$

Herein, we examined the feasibility of using RMS to analyze molecular changes during $T$. gondii infection at a single cell level. We dissect the molecular interaction between $T$. gondii infection and human cells based on spatially- and time-resolved RMS. Human retinal cells were infected with $T$. gondii tachyzoites and Raman spectra were measured from the parasites and the surrounding cytoplasm of the host cells. Initial experiments 
were performed on paraformaldehyde-fixed cells, to provide an overview of the molecular changes, followed by measurements on live cells to demonstrate the potential of Raman microscopy for label-free time-course analysis. Our findings reveal previously unrecognized chemical features of $T$. gondii tachyzoites interacting with host cells and suggest that these biomolecules contribute to $T$. gondii invasion and parasite virulence during infection.

\section{Materials and Methods}

\section{Cell culture}

The human retinal pigment epithelial cell line (ARPE-19) was kindly provided by Dr Kevin Webb and Emilia Moradi, University Of Nottingham. Cells were maintained in vitro in Dulbecco's modified Eagle's medium, low glucose, pyruvate (DMEM, Life technologies, Grand Island, NY) supplemented with $1 \%$ penicillin/streptomycin (Invitrogen Corp., Carlsbad, CA) and 10\% heat-inactivated fetal bovine serum (FBS, Invitrogen Corp., Carlsbad, CA). As an immune-privileged and easily accessible organ, the eye constitutes a favourable target for $T$. gondii. Hence, we selected this cell line as a model to examine the injury caused by $T$. gondii infection to the eye.

\section{Growth and preparation of parasites}

Toxoplasma gondii tachyzoites were maintained in vitro by serial passage on monolayers of human brain microvascular endothelial cells (HBMECs) at $37^{\circ} \mathrm{C}$ in $5 \% \mathrm{CO} 2$. HBMECs were grown as described previously. ${ }^{19}$ Infected host cell monolayers were scraped, parasites were isolated from host cells by passage through 25- and 27-gauge needles and purified by using PD-10 Desalting Columns prepacked with Sephadex G-25 medium as described previously. ${ }^{20}$ Purified parasites were centrifuged at $800 \times \mathrm{g}$, washed twice with fresh cRPMI, re-suspended in fresh medium and quantified using a hemacytometer. The final volume of suspension was adjusted with cRPMI medium to achieve a ratio of $2: 1$ parasite/host cell for subsequent infection experiments. Parasite viability was checked by using trypan blue staining assay and parasite with more than $97 \%$ viability were used.

\section{In vitro infection protocol}

ARPE-19 cells $\left(3 \times 10^{5}\right.$ cells $\left./ \mathrm{mL}\right)$ were seeded in titanium cell-chambers that fitted 6well culture plates with a volume of $2 \mathrm{~mL}$ cRPMI medium/well. The titanium cell chambers were purpose-built to enable acquisition of Raman spectra of the cells and incorporated $\mathrm{MgF} 2$ coverslips ( $0.17 \mathrm{~mm}$ thick) at the bottom. The Cells were allowed to grow overnight by incubation at $37^{\circ} \mathrm{C}$ in a humidified atmosphere with $5 \% \mathrm{CO} 2$ in air. Before infection, cell growth medium was removed and cells were washed three times with sterile PBS (8 g/L NaCl, $0.2 \mathrm{~g} / \mathrm{L} \mathrm{KCl}, 0.2 \mathrm{~g} / \mathrm{L} \mathrm{KH} 2 \mathrm{PO} 4,1.15 \mathrm{~g} / \mathrm{L} \mathrm{Na} 2 \mathrm{HPO} 4)$. Then, in each chamber parasites were added at a MOI of 2 in 2-ml fresh medium, and the control chambers received only 2-ml fresh medium (mock-infected). Culture plates were then incubated to allow infection to progress within cells. Parasite and control cultures were sampled at different time point post infection (PI) starting from $0 \mathrm{~h}$, and then, at 6 , 24 and $48 \mathrm{~h}$ PI. At each sampling time two chambers (one infected and one control) were collected from the 6-well culture plate and subjected to Raman Spectroscopy imaging. 


\section{Raman spectroscopy}

Raman spectra were recorded using a home-built confocal Raman micro-spectrometer optimized for live-cell studies (Supplementary Information Figure S1). ${ }^{21}$ An inverted microscope (IX71, Olympus, Essex, UK) was chosen for the setup as it allows live cell measurements without additional disturbance of objective dipping in culture media as opposed to up-right microscopes. The laser is focused through a $\mathrm{MgF}_{2}$ coverslip at the bottom of specially designed sample holders. An environmental enclosure (Solent, Segensworth, UK) was integrated to the microscope to ensure suitable physiological conditions for living cells, $37^{\circ} \mathrm{C}$ temperature and $5 \% \mathrm{CO}_{2}$. Under these conditions, Raman spectral measurements of individual live cells maintained in their original culture media were obtained over periods of several hours without disturbing the cells and also avoiding bacterial contamination. A $785 \mathrm{~nm} \sim 200 \mathrm{~mW}$ laser (before objective) (Ti:sapphire laser, Spectra-Physics) was used for excitation of Raman spectra. To maximise the spatial resolution and collection efficiency for the Raman spectra, a waterimmersion 60x objective, NA 1.20 (Olympus) was used for focusing the laser (beam diameter expanded to match the objective pupil, laser spot $\sim 700 \mathrm{~nm}$ on sample) on individual cells as well as for collection of Raman scattered photons. The Raman scattered light was collimated and then focused on a $50 \mu \mathrm{m}$ diameter optical fibre connected to a spectrometer equipped with a 830 lines/mm grating (spectral resolution of $\sim 1.5 \mathrm{~cm}^{-1}$ in the $600-1800 \mathrm{~cm}^{-1}$ region) and cooled deep-depletion back-illuminated CCD detector (Andor Technologies, Belfast, UK). Raman spectral imaging was performed by scanning areas of the cells through the laser focus in a raster pattern $(1 \mu \mathrm{m}$ step size, typically areas of $15 \mu \mathrm{m} \times 15 \mu \mathrm{m}$ at 6 hours PI up to $40 \mu \mathrm{m} \times 60 \mu \mathrm{m}$ at 48 hours PI) using a high-precision step-motor stage (Prior, Cambridge, UK) and acquiring Raman spectra at each position ( $2 \mathrm{~s} /$ pixel). The spectrometer wavenumber axis was calibrated prior to each experiment using tylenol at an accuracy of $0.5 \mathrm{~cm}^{-1}$.

\section{Fluorescence imaging}

Morphological changes in the development of $T$. gondii tachyzoites within host cells were studied by the acridine orange (AO) staining. AO is a DNA intercalating dye and is used to differentially stain RNA and DNA inside eukaryotic cells. Cells were fixed in 4\% paraformaldehyde for $15 \mathrm{~min}$, followed by rinsing in 1x PBS twice. Then, fixed cells were incubated with AO stain for approximately $10 \mathrm{~min}$. AO fluorescence imaging of $T$. gondii-infected cells was performed on the same cells after completion of the Raman spectral imaging experiment. Wide-field fluorescence imaging was integrated on the confocal Raman micro-spectrometer. The retrospective positioning and identification of the cells was based on two thin marks engraved on the cell chambers (retro-positioning accuracy was $5 \mu \mathrm{m})$.

\section{Data analysis}

Data pre-processing and analysis was done by using in-house built functions in Matlab (The MathWorks, Natick, MA). The spectra were collected from cytoplasm of control and cells and areas surrounding parasites in the infected cells. Data pre-processing involved cosmic rays removal, background subtraction and normalisation of the spectra. The cosmic ray removal algorithm was designed to detect the gradient in a Raman spectrum. If for a peak in the spectrum the absolut value of the gradient was found larger 
than the average gradient in the Raman spectra of all cells plus $10 \times$ standard deviations, the peak was considered to be a cosmic ray peak. Once, detected, the algorithm removed seven data points, and replaced them with points using linear interpolation. For each cells, a background spectrum was calculated as the mean of 100 Raman spectrea measured in a $10 \mu \mathrm{m} \times 10 \mu \mathrm{m}$ grid in a region near the cell. For each cell, this background spectrum was subtracted. All spectra were normalised to zero mean and unit variance. After pre-processing, Raman images obtained by mapping the scores for PC2 were overlapped with fluorescent images using image registration in Matlab. After this, the spectra were selected manually from regions of the cytoplasm or parasites. The resulting Raman spectra of fixed cells were then analysed by principal component analysis (PCA); this model was then applied to calculate the PCA scores for the Raman spectra corresponding to live cells. The PC2 scores for for all spectra were analysed by Student ttest.

For the Raman spectra presented in Figures 1, 3, 4, 5, and 7, a noise reduction algorithm was applied based on PCA, in which all Raman spectra were reconstructed based on the first $10 \mathrm{PCs}{ }^{5,7}$.

\section{Results and Discussion}

\section{Raman spectra of human retinal cells and $T$. gondii}

Figure 1 shows typical examples of Raman spectra and molecular images for healthy human retinal cells and isolated $T$. gondii. For the retinal cells, fluorescence images corresponding to acridine orange (AO) staining have been acquired after the completion of the Raman spectroscopy measurements. The stain was taken up by both parasiteinfected and control cells. 
a

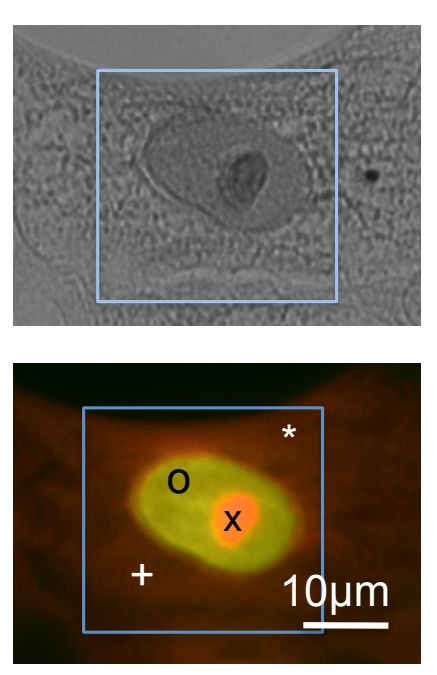

b

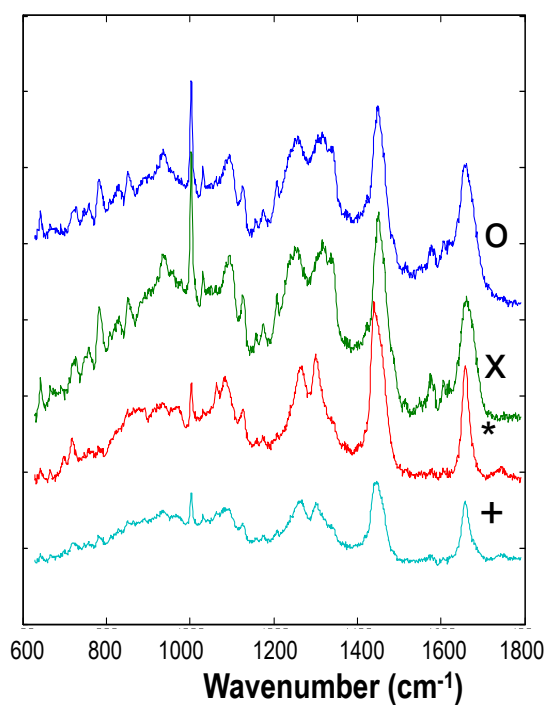

d
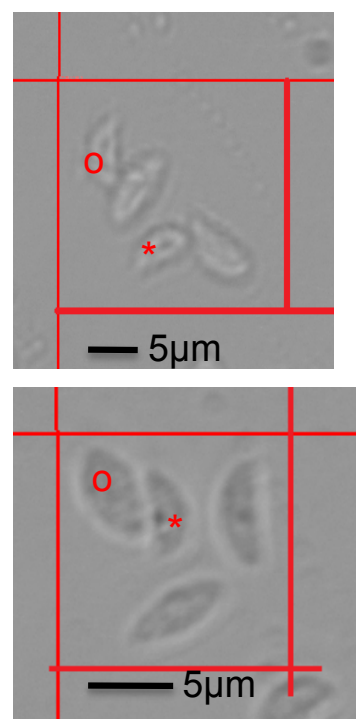

e

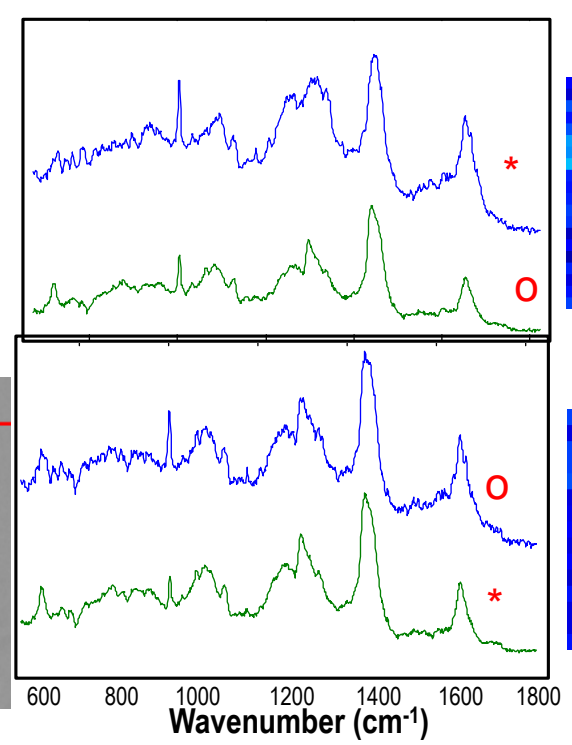

C
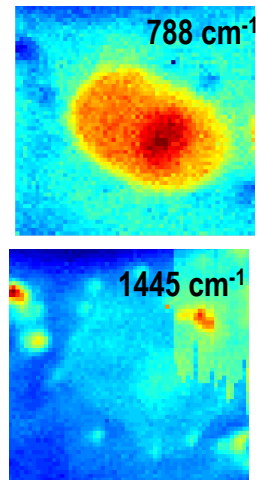

$1005 \mathrm{~cm}^{-1}$

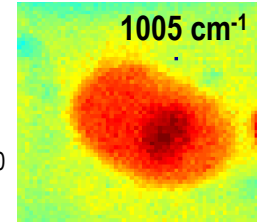

f

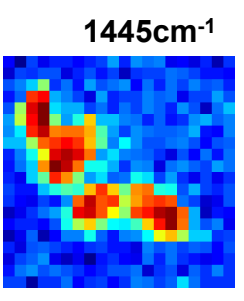

$1445 \mathrm{~cm}^{-1}$

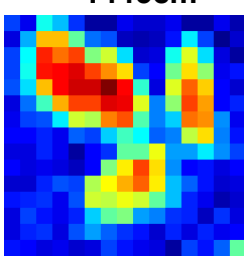

Fig. 1. Raman spectra and label-free molecular imaging of healthy human retinal cells and isolated $T$. gondii tachyzoites. a) Brightfield (top panel) and acridine orange fluorescence (bottom panel) images of a typical retinal cell. Fluorescence images are obtained after completion of the Raman spectroscopy measurements. b) Typical examples of Raman spectra acquired at different locations inside the cell. c) Raman images obtained using the peak intensity of the $788 \mathrm{~cm}^{-1}$ (nucleic acids), $1445 \mathrm{~cm}^{-1}$ (lipids \& proteins), and $1005 \mathrm{~cm}^{-1}$ (proteins) bands. d) Bright field image of isolated $T$. gondii. e) Selected Raman spectra of $T$. gondii tachyzoites. f) Raman images obtained using the peak intensity of the $1445 \mathrm{~cm}^{-1}$ band (lipids \& proteins). 
Fig. 1b shows that the Raman spectra measured from the cell nuclei elicit intense bands corresponding to nucleic acids (O-P-O $788 \mathrm{~cm}^{-1}, \mathrm{PO}_{2}^{-} 1098 \mathrm{~cm}^{-1}$, ring vibrations of $\mathrm{G}$ and A $1578 \mathrm{~cm}^{-1}$ ) and proteins (phenylalanine $1005 \mathrm{~cm}^{-1}$, tyrosine $830 \mathrm{~cm}^{-1}$ and $850 \mathrm{~cm}^{-1}$, amide I $1660 \mathrm{~cm}^{-1}$, amide III 1200-1400 $\mathrm{cm}^{-1}, \mathrm{CH}_{2}$ deformation $1449 \mathrm{~cm}^{-1}$ ), with the highest intensity measured in the nucleolus.

Bands corresponding to proteins are also present in the Raman spectra of cytoplasm, but the most intense bands are assigned to lipids: $\mathrm{C}=\mathrm{C}$ stretching $1660 \mathrm{~cm}^{-1}, \mathrm{CH}_{2}$ deformation $1445 \mathrm{~cm}^{-1}, \mathrm{CH}_{2}$ twisting $1303 \mathrm{~cm}^{-1}$, =C-H deformations $1267 \mathrm{~cm}^{-1}$, O-P-O symmetrical stretch and acyl back bone C-C bands at $1090 \mathrm{~cm}^{-1}$ and $1062 \mathrm{~cm}^{-1}$, symmetric stretching of $\mathrm{N}\left(\mathrm{CH}_{3}\right)_{3}$ of choline group $719 \mathrm{~cm}^{-1}$. Micrometric regions rich in lipids can be identified in the cytoplasm, corresponding probably to lipid bodies. The Raman spectra of isolated fixed T. gondii (Figs. 1d,e) are dominated by intense bands corresponding to molecular vibrations of lipids and proteins. The spectra of $T$. gondii have higher intensity-ratio between the $\mathrm{CH}_{2}$ twisting $1303 \mathrm{~cm}^{-1}$ and $=\mathrm{C}-\mathrm{H}$ deformations $1267 \mathrm{~cm}^{-1}$ bands compared to healthy retinal cells, suggesting a higher concentration of saturated lipids in $T$. gondii. It is also interesting to note that only small contribution from nucleic acids was detected in the Raman spectra of isolated T. gondii.
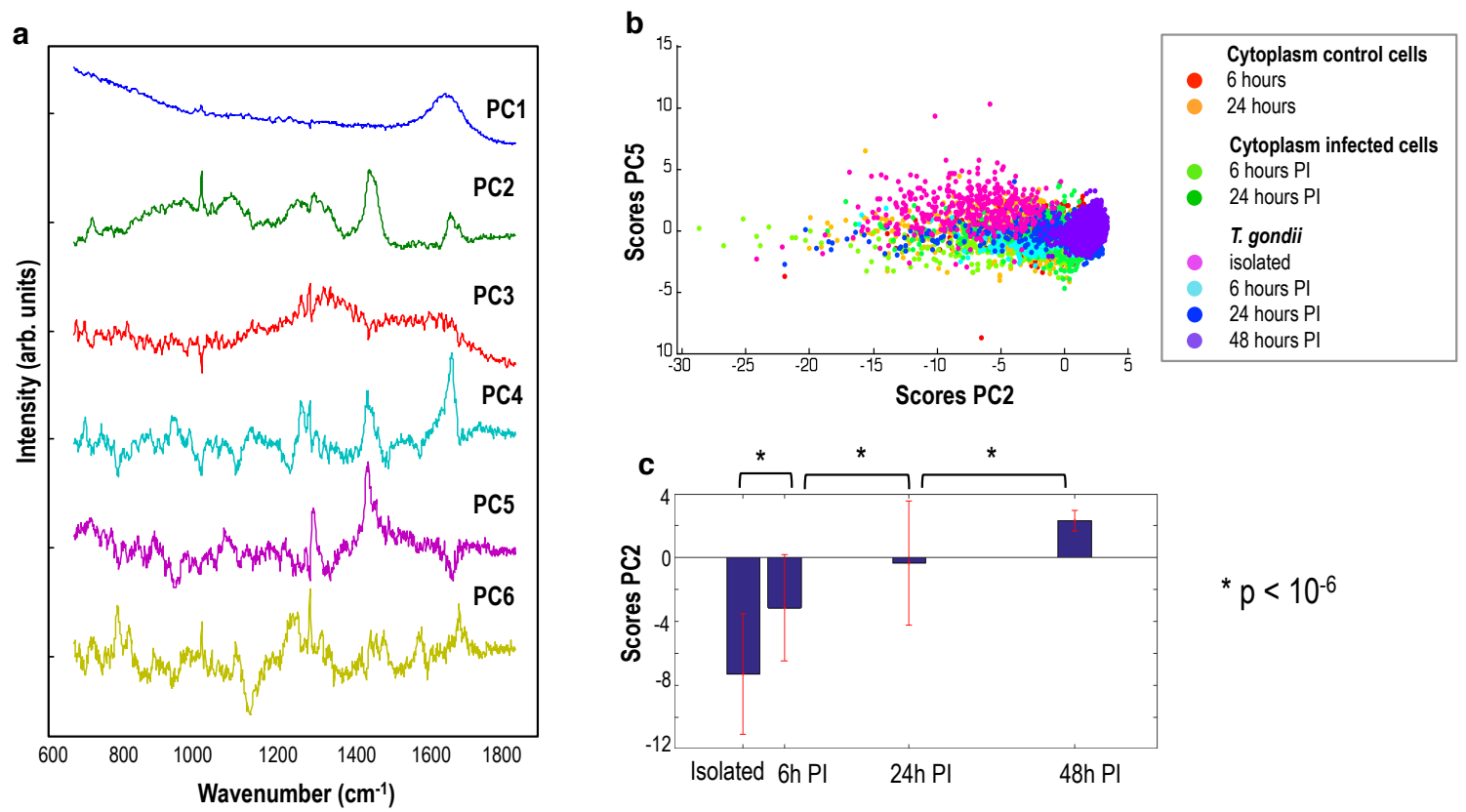

Fig. 2. Principal component analysis (PCA) of the Raman spectra collected from control human retinal pigment epithelial cell line (ARPE-19) cells, isolated $T$. gondii parasites, $T$. gondii inside ARPE-19 host cells (6 hr, $24 \mathrm{hr}, 48 \mathrm{hr}$ PI), cytoplasm of the ARPE-19 cells in the vicinity of the $T$. gondii $(6 \mathrm{hr}, 24 \mathrm{hr}$ PI). a) PCA loadings. b) scatter plot PC2 vs PC5 for all spectra; c) PC2 scores of isolated T. gondii and T. gondii in host cells, * represents $\mathrm{p}<10^{-6}$ for t-test, error bars represent standard deviations.

Time-course study of pathogen-host interaction using fixed cells in parallel cultures 
One of the key challenges when measuring Raman spectral maps of live cells is the potential changes in cell morphology during the typically long acquisition times of rasterscanning (20-40 min). Such morphological changes make it difficult to compare the Raman maps with images obtained by other label-based modalities in order to identify the exact locations in a cell where the spectra were measured. Thus, prior to performing time-course experiments on live cells, Raman spectroscopy mapping by raster-scanning was carried out on cells fixed with $4 \%$ ice-cold paraformaldehyde. After Raman mapping, the cells were stained with acridine orange, allowing identification of the $T$. gondii growing in the retinal host cells (red emission for RNA mainly in the cytoplasm and green emission for DNA in the nuclei). Measurements were carried out on cytoplasmic regions of control (non-infected) human retinal cells and from $T$. gondii tachyzoites within the host cells at $6 \mathrm{hr}, 24 \mathrm{hr}$ and $48 \mathrm{hr}$ post infection (PI). Ten cells were measured at each type point. To provide a statistical analysis of the Raman spectra that would account for both intra- and inter-cellular variability, all spectra were included into a single dataset and were analysed by principal component analysis (PCA).

a

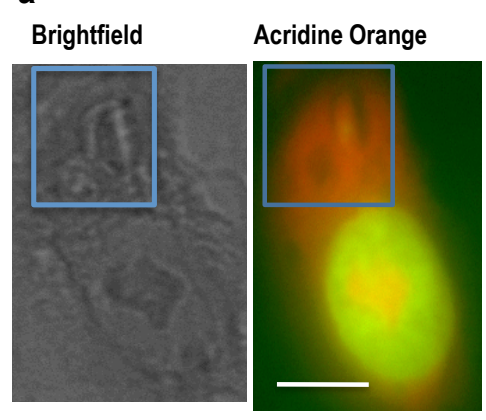

b
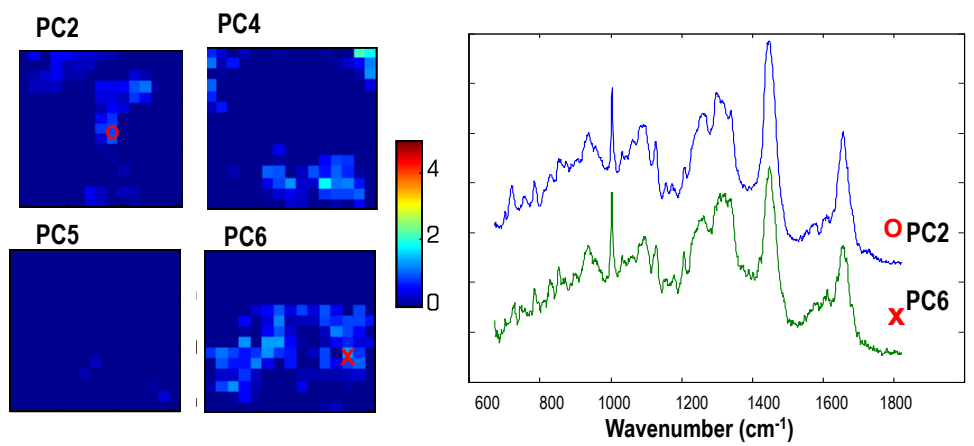

PC2

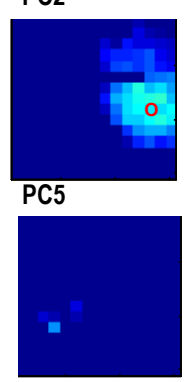

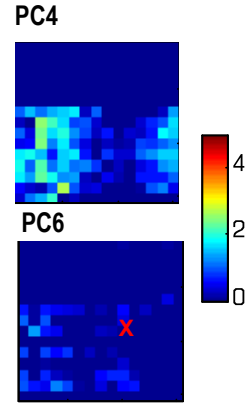

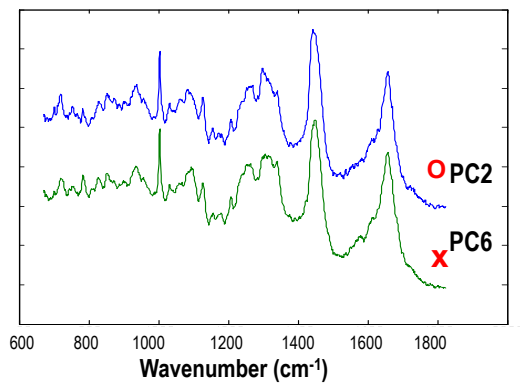

Fig. 3. Raman analysis of $T$. gondii infected cells at $6 \mathrm{hr}$ PI. a) Bright field and fluorescence images (recorded after Raman spectroscopy measurements). b) Maps corresponding to principal components PC2, PC4, PC5 and PC6. c) Selected Raman spectra at locations indicated by o symbols. Scale bars: 10 microns.

Fig. 2a presents the loadings of the first six principal components accounting to $58 \%$ of the total variance of the spectra (component seven was dominated by noise). While the loadings corresponding to PC1 and PC3 consisted only of broad bands characteristic to the background water, the loadings corresponding to PC2, PC4, PC5 and PC6 consisted of bands that can be correlated to specific molecular vibration in biomolecules. PC2 
( $14.88 \%$ variance) is dominated by strong bands corresponding to proteins and lipids. PC4 (2.20\% variance) and PC 5 (1.18\% variance) have bands mostly associated to lipids, while PC6 $\left(0.79 \%\right.$ of variance) has positive bands at $788 \mathrm{~cm}^{-1}$ and $813 \mathrm{~cm}^{-1}$ that can be associated to nucleic acids.

The increase in PC2 scores with the infection time indicates an increase in protein and lipid amount in $T$. gondii during infection. Inspection of the scores related to the other principle components indicates that these components capture only inter- and intracellular variability related to lipids and nucleic acids, but no trends related to $T$. gondii infection can be observed. Figure $2 \mathrm{~b}$ presents 2 -D plots corresponding to the principal component scores. The results show that PC2 captures most spectral changes related to the T. gondii developing inside the host ARPE-19 retinal cells. Although the PC2 scores for the Raman spectra of isolated $T$. gondii tachyzoites and cytoplasm regions of the healthy retinal cells (control) vary within a broad range reflecting the inter- and intracellular variability, Figure 2c shows that the PC2 scores corresponding to the spectra of T. gondii increase as soon as $6 \mathrm{hr}$ PI to form a distinct cluster at $48 \mathrm{hr}\left(\mathrm{p}<10^{-6}\right.$ in t-test). .

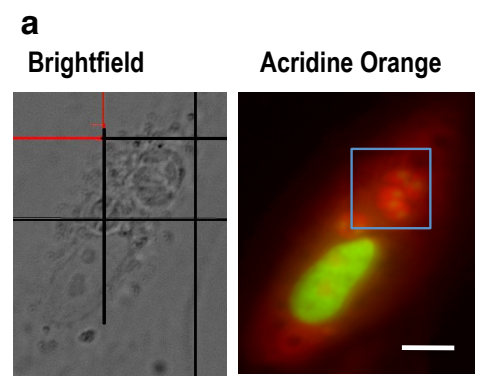

b
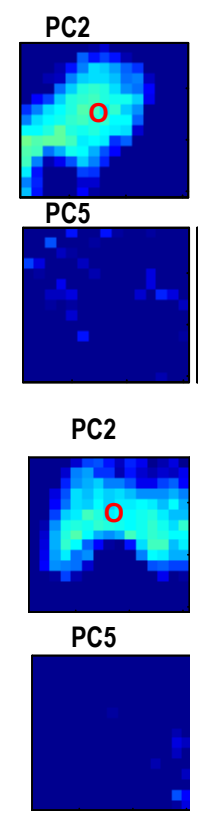

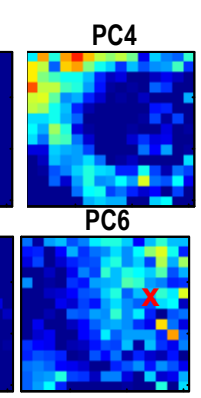

PC4

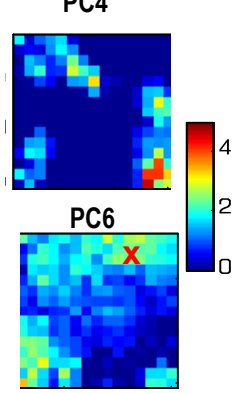

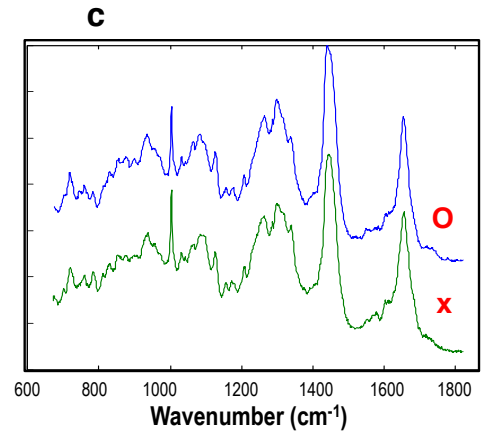

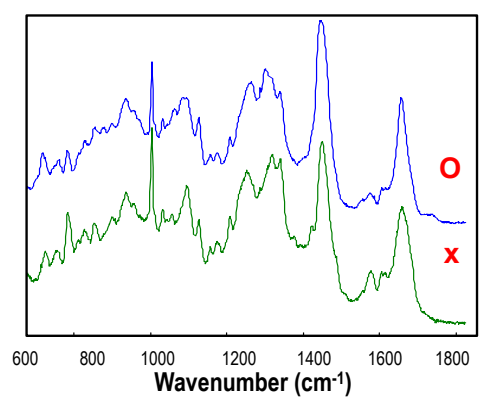

Fig. 4. Raman analysis of $T$. gondii infected cells at $24 \mathrm{hr}$ PI. a) Bright field and fluorescence images (recorded after Raman spectroscopy measurements). b) Maps corresponding to principal components PC2, PC4, PC5 and PC6. c) Selected Raman spectra at locations indicated by o symbols. Scale bars: 10 microns.

To obtain a better understanding of the molecular changes captured the PCA, Raman maps corresponding to the principal components scores were created. Fig. 3a shows that at $6 \mathrm{hr}$ after the addition of $T$. gondii tachyzoites to the retinal cell cultures, typically only 1-2 parasite cells were found inside the host cells. The PC2 maps indicate higher scores at the locations of the $T$. gondii and the Raman spectra measured at these locations, indeed, show intense bands assigned to lipids and proteins. The Raman spectra of T. gondii inside 
the host cells also show bands corresponding to nucleic acids $\left(788 \mathrm{~cm}^{-1}\right)$, bands which were not detected in the isolated $T$. gondii tachyzoites. These changes may correlate to the increase expression of RNA required for protein synthesis during the growth of the $T$. gondii. After $24 \mathrm{hr} \mathrm{PI}$, the $T$. gondii tachyzoites multiplied inside the host cells and typically 3-4 parasites can be observed in the bright field and fluorescence images. The scores corresponding to the PC2 increases compared to $6 \mathrm{hr}$ and the Raman spectra indicate intense bands assigned to lipids and proteins. The maps corresponding to the PC6 also show an increase of nucleic acids.

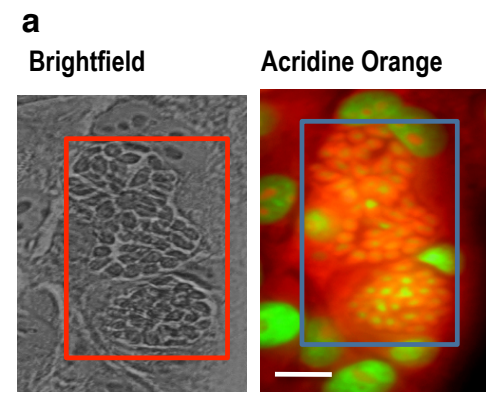

b
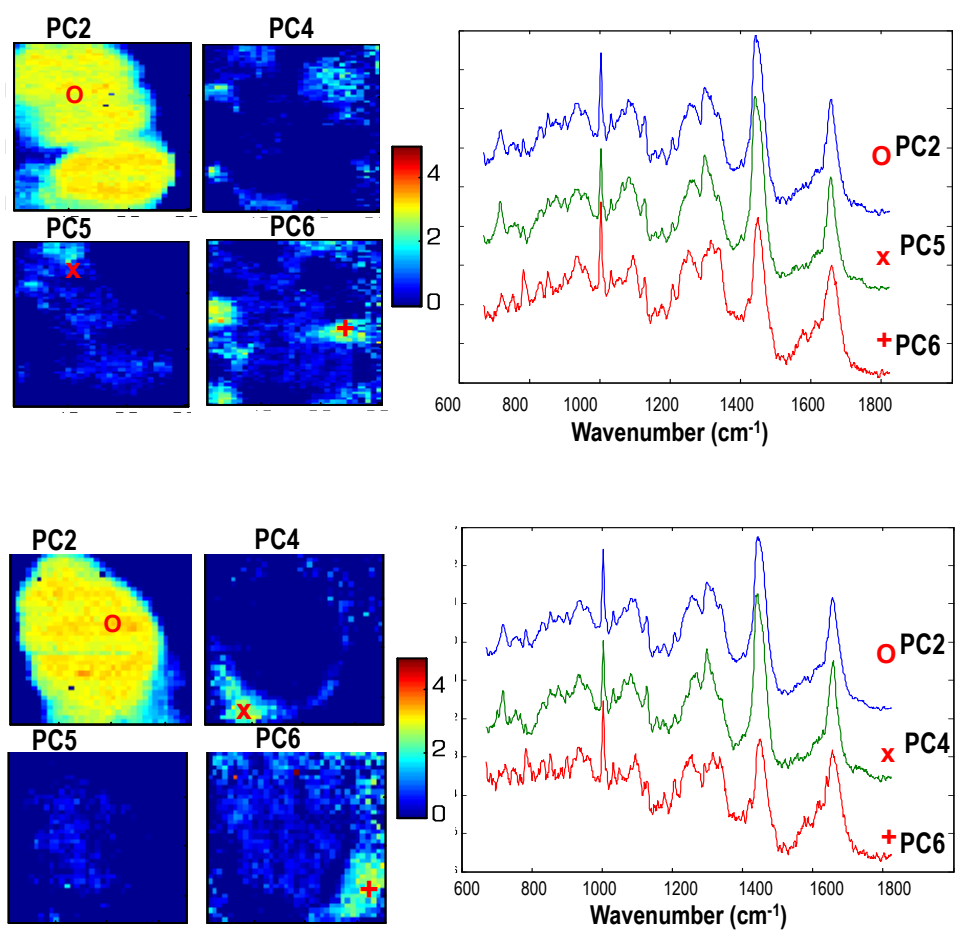

Fig. 5. Raman analysis of $T$. gondii infected cells at $48 \mathrm{hr}$ PI. a) Bright field and fluorescence images (recorded after Raman spectroscopy measurements). b) Maps corresponding to principal components PC2, PC4, PC5 and PC6. c) Selected Raman spectra at locations indicated by o symbols. Scale bars: 10 microns.

At $48 \mathrm{hr}$ PI, T. gondii formed a large vacuole consisting of more than 10 tachyzoites that occupied almost the entire cytoplasm of the host cells. The nuclei of the host cells are squeezed at the edges of the cells. The PC2 scores reached the highest values indicating the increase in protein and lipid synthesis during infection. The maps corresponding to the PC6 scores correlate to the locations of the nuclei of both host and T. gondii tachyzoites, however the intensity of the $788 \mathrm{~cm}^{-1}$ and $1098 \mathrm{~cm}^{-1}$ bands are similar to the intensities at $6 \mathrm{hr}$ and $24 \mathrm{hr}$ PI. In addition, the PC4 maps at $48 \mathrm{hr}$ PI show an increase in the PC4 scores in the host cytoplasm surrounding the parasite vacuole, suggesting an increased concentration of saturated lipids. Selected Raman spectra at these locations indicate an increase in the intensity ratio of the $1303 \mathrm{~cm}^{-1}$ and $1273 \mathrm{~cm}^{-1}$ bands. 


\section{Raman spectroscopy of live $T$. gondii during infection}

After establishing the PCA model based on the time-course Raman spectral maps measured from paraformaldehyde-fixed cells, the model was applied to new Raman spectral datasets measured from live cells. Human retinal cells were cultured in titanium cell-chambers, and after the addition of live $T$. gondii tachyzoites, the cell chambers were placed on the Raman microscope in the environmental enclosure for a period of $48 \mathrm{hr}$ (additional cell-chambers with control healthy retinal cells were also included). Fig. 6 shows the scores corresponding to PC2, PC4, PC5 and PC6 (calculated using the model developed for the fixed cells) for the live $T$. gondii-infected retinal cells. While the scores for PC4, PC5 and PC6 showed no spectral differences between the control live and fixed cells, the PC2 scores for the live $T$. gondii tachyzoites matched the scores for the fixed $T$. gondii at $48 \mathrm{hr}$ PI. The results agree with the bright field and AO images (Fig. 7a) that showed the parasite vacuoles contained 6-10 parasites growing in the cytoplasm of the host cells. The PC2 maps show good correlation with the AO images and the selected Raman spectra indicated strong bands corresponding to proteins and lipids.

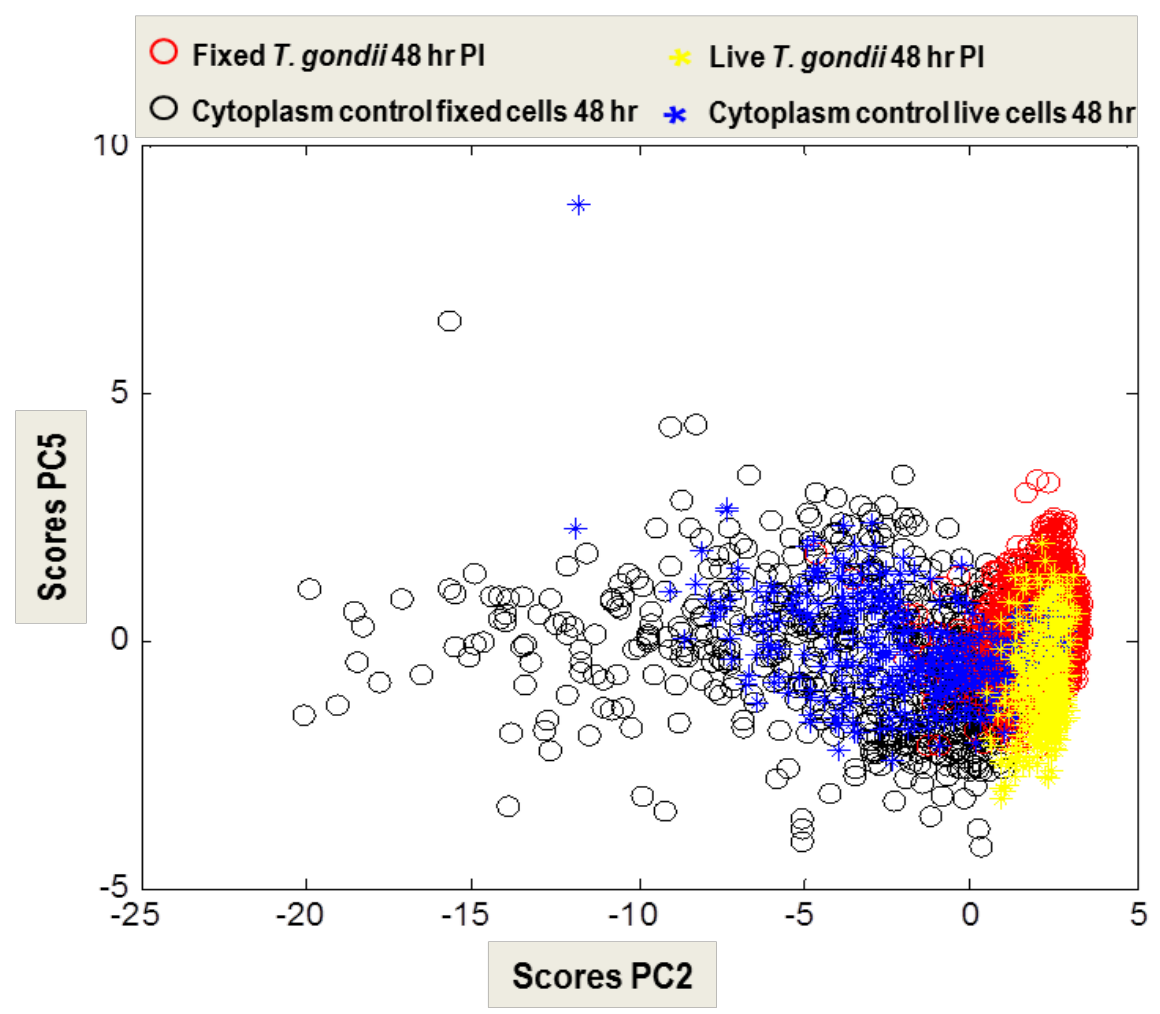

Fig. 6. PCA analysis of the Raman spectra for live $T$. gondii tachyzoites inside ARPE-19 cells after $48 \mathrm{hr}$ PI. The scores corresponding to PC2 and PC5 were calculated using the model developed for fixed cells (Fig. 2).

Lipid droplets, major lipid storage structures, are composed of a triglyceride and cholesteryl ester core with a surrounding monolayer of phospholipid, cholesterol, and a 
variety of associated proteins with diverse functions in cell metabolism, signaling, and inflammation. ${ }^{22-23}$ The association between $T$. gondii infection and increased concentrations of proteins and lipids is consistent with a previous report where the size and number of lipid droplets, whose major component is TGAs, increased in cells infected by $T$. gondii. ${ }^{24}$ The interaction of parasite proteins with these lipid bodies is important for the replication of intracellular pathogen, such as $T$. gondii and for the biogenesis of new parasite particles. ${ }^{24}$ The intense bands at $788 \mathrm{~cm}^{-1}$ also indicated increased amount of nucleic acids, also confirmed by the intense AO fluorescence intensity. The source of the nucleic acids' traces, host or parasite origin, is unknown. However, the concentration of nucleic acids was found to increase in the infected cultures in proportion to the proliferation of parasite, suggesting the source of the nucleic acids to be of parasite origin. This finding is consistent with a recently reported finding in the related protozoan $N$. caninum. ${ }^{25}$

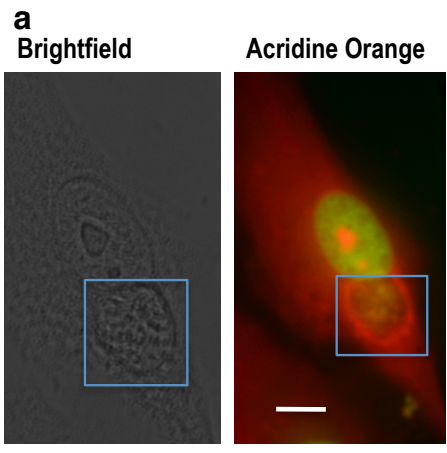

b
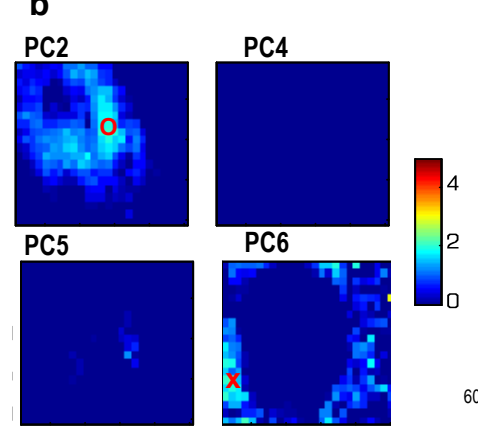

C
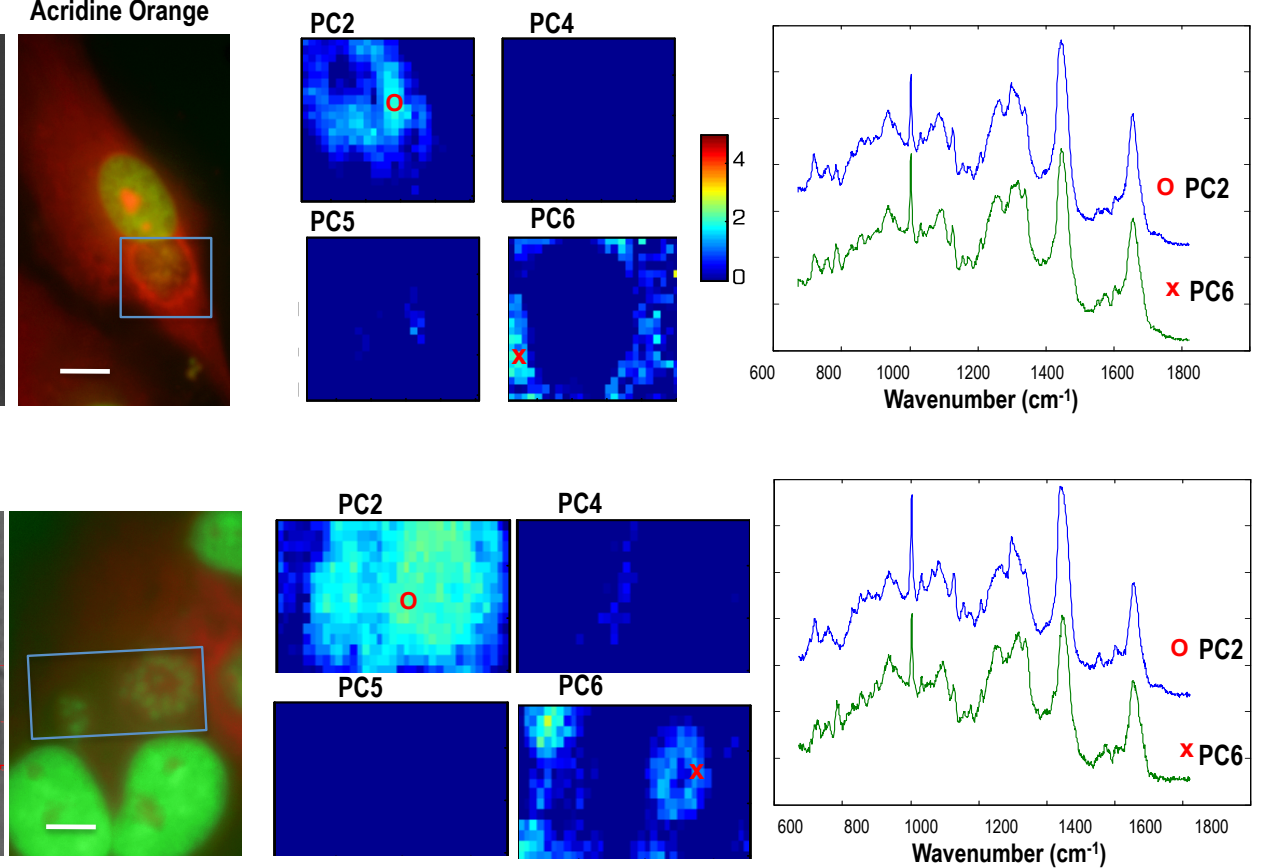

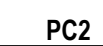

PC4

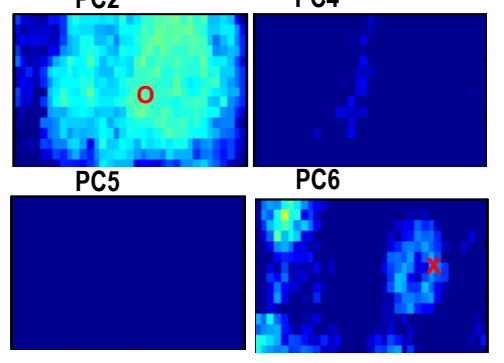

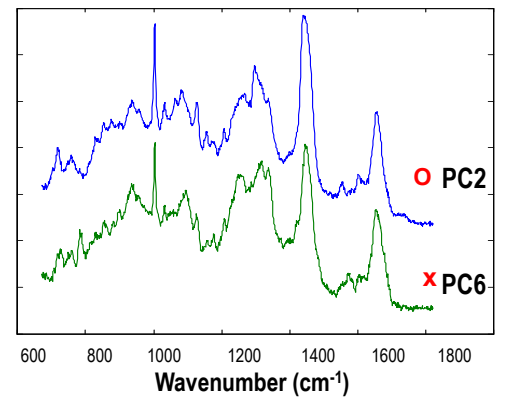

Fig. 7. Live ARPE-19 cells infected with T. gondii at $48 \mathrm{hr}$ PI. a) Bright field and fluorescence images (recorded after Raman spectroscopy measurements). b) Maps corresponding to principal components PC2, PC4, PC5 and PC6. c) Selected Raman spectra at locations indicated by o symbols. Scale bars: $10 \mu \mathrm{m}$.

The application of RMS in studying host-pathogen interaction in the field of parasitology is promising. Raman spectroscopy was successfully used to compare healthy erythrocytes and erythrocytes infected with Plasmodium falciparum. ${ }^{16}$ RMS was also used to monitor molecular changes in red blood cells during Plasmodium infection in mice, following malaria disease progression over the course of 7 days. ${ }^{17}$ Changes in the Raman spectra of cells was associated with the loss of hemoglobin and increase of hemozoin 4 days PI. ${ }^{17}$ In the present study, we report the first study using RMS to investigate the infection of human retinal cells by protozoan pathogens. The use of ARPE-19 cells is relevant and 
has medical importance because this cell line can be used as a model of blood-retinal barrier, which is fundamentally important for the protection of the eye and for maintenance of vital ocular functions.

The challenges that $T$. gondii-infected humans and animals face with regard to ocular complications are prominent. In humans, $T$. gondii is most frequently involved in the causation of infectious uveitis. ${ }^{2}$ In dogs and cats $T$. gondii is a significant cause of ocular morbidity including inflammation and scarring of the retina (retinitis), iris (uveitis) and cornea (keratitis), resulting in severe pain and welfare implications. Our crossdisciplinary study combines cell-based integrated imaging modalities that allowed us, for the first time, to uncover $T$. gondii-associated functional alterations in the ARPE-19 cells. Establishment of unique phenotypic and chemical profiles of human retinal pigment epithelial cells (ARPE-19) specific to T. gondii infection will lead to identification of host pathways with pharmacological potential for drug discovery.

\section{Conclusions}

Raman micro-spectroscopy was used to measure label-free molecular properties of $T$. gondii- infected host cells. Significant increase in protein and lipid concentration was reported associated with development of infection as confirmed by time-course experiments on both paraformaldehyde-fixed and live infected cells. The development of $T$. gondii cells was also accompanied by an increase in nucleic acids as early as $6 \mathrm{hr}$ PI. While this study demonstrates the potential of Raman spectroscopy to detect molecular changes during host-pathogen interaction, the current method does not provide sufficient specificity to pinpoint the main proteins or lipids that are affected during this biological process. Further studies are currently planned using isotope labelled molecules to improve the specificity and discrimination between biomolecules originating from the $T$. gondii and host cells. These future experiments are likely to provide further information related to molecular exchange between pathogen and host cells. Understanding the timeand spatially-dependent molecular interactions between pathogens and host cells may provide a useful platform for screening compounds with potential neuro-protective activity for eminent effects of changes in brain infection control practices.

\section{Acknowledgments}

The work was supported by Petplan Charitable Trust grant 11-38 (H. Elsheikha and I. Notingher). The development of the Raman micro-spectrometer was supported by the Biotechnology and Biological Sciences Research Council UK research grant BB/G010285/1. A. Naemata acknowledges the support from COMSATS Institute of Information Technology, Pakistan.

1. H. M. Elsheikha, Public Health, 2008, 122(4), 335-353.

2. C. Hooper, P. McCluskey, Curr. Allergy Asthma Rep., 2008, 8, 331-338.

3. K. E. McGovern, E. H. Wilson, Parasit Vectors., 2013, 6(1), 334.

4. I. Notingher, S. Verrier, S. Haque, J. M. Polak, L. L. Hench, Biopolymers 2003, 72 (4), 230-240 
5. N. Uzunbajakava, A. Lenferink, Y. Kraan, E. Volokhina, G. Vrensen, J. Greve, C. Otto, Biophys. J., 2003, 84(6), 3968-3981.

6. C. Matthäus, T. Chernenko, J. A. Newmark, C. M. Warner, M. Diem, Biophys. J., 2007, 93, 668-673.

7. A. Ghita, P. C. Flavius, M. Mather, V. Sottile, I. Notingher, Anal. Chem., 2012, 84(7), 3155-3162

8. C. Matthäus, C. Krafft, B. Dietzek, B. R. Brehm, S. Lorkowski, J. Popp, Anal Chem. 2012 84(20), 8549-8556.

9. S. H. Eder, A. M. Gigler, M. Hanzlik, M. Winklhofer, PLoS One., 2014, 9(9), e107356.

10. Y. S. Huang, T. Karashima, M. Yamamoto, H. O. Hamaguchi, Biochemistry, 2005, 44, 10009-10019.

11. A. Zoladek, F. C. Pascut, P. Patel, I. Notingher, J. Raman Spectrosc., 2011, 42(3), 251-258.

12. M. Okada, N. I. Smith, A. F. Palonpon, H. Endo, S. Kawata, M. Sodeoka, K. Fujita, Proc. Natl. Acad. Sci. U S A., 2012, 109(1), 28-32.

13. H. N. Noothalapati Venkata, S. Shigeto, Chem. Biol., 2012, 19, 1373-1380.

14. F. C. Pascut, S. Kalra, V. George, N. Welch, C. Denning, I. Notingher, Biochim Biophys Acta., 2013, 1830(6), 3517-3524.

15. A. Ghita, F. C. Pascut, V. Sottile, I. Notingher, Analyst, 2014,139, 55-58.

16. B. R. Wood, S. J. Langford, B. M. Cooke, F. K. Glenister, J. Lim, D. McNaughton, FEBS Lett.,2003, 554(3), 247-252.

17. A. J. Hobro, A. Konishi, C. Coban, N. I. Smith, Analyst., 2013, 138(14), 3927-3933.

18. K. Kong, C. J. Rowlands, H. Elsheikha, I. Notingher, Analyst., 2012, 137(18), 4119 4122.

19. H. M. Elsheikha, C. L. McKinlay, N. A. Elsaied, P. A. Smith, Parasit. Vectors., 2013, 6, 24.

20. H. M. Elsheikha, B. M. Rosenthal, A. J. Murphy, D. B. Dunams, D. A. Neelis, L. S. Mansfield, Vet. Parasitol., 2006, 135, 223-234.

21. F. C. Pascut, H. T. Goh, N. Welch, L. D. Buttery, C. Denning, I. Notingher, Biophys J., 2011, 100(1), 251-259.

22. P. T. Bozza, H. D’Avila, P. E. Almeida, K. G. Magalhães, R. Molinaro, C. J. Almeida, C. M. Maya-Monteiro, Clin. Lipidol., 2009, 4, 791-807.

23. R. V. Jr. Farese, T. C. Walther, Cell, 2009, 139, 855-860.

24. R. C. N. Melo, D. L. Fabrino, F. F. Dias, G. G. Parreira, Inflamm. Res., 2006, 55, 342-348.

25. H. M. Elsheikha, M. Alkurashi, K. Kong, X. Q. Zhu, BMC Res. Notes, 2014, 7,406. 